Seiten bestehenden Unzufriedenheit eine Kompromisslösung ausloten wolle und einen zweiten Arbeitsentwurf angekündigt habe.

Im Rahmen der darauf folgenden Diskussion befassten sich die Teilnehmer in erster Linie mit ordnungspolitischen Fragen, die sich in Folge des Vortrages stellten. Hierbei wurden, insbesondere von Frank Andreas Schendel, auch Vergleiche $\mathrm{zu}$ anderen privatisierten Sektoren angestellt. Besonders heiß diskutiert wurde die Frage, wem der Zugriff auf die Wertstoffströme zweckmäßigerweise zugeordnet werden solle - den Kommunen oder den Privaten. Wolfgang Durner hob dazu hervor, dass dies aus einer ökologischen Perspektive unbedeutend sei und der Streit um die Zuständigkeiten von finanziellen Erwägungen der Akteure befeuert werde.
Gruneberg vertrat dagegen den Standpunkt, die Kommune müsse Zugriff auf die Wertstoffströme haben, um ihrer kommunalen Verantwortung gerecht zu werden - eine These, die von Michael Scheier angegriffen wurde, jedoch von Georg Wulf mit Blick auf die kommunale Selbstverwaltungsgarantie differenziert betrachtet wurde.

Darüber hinaus fand im Rahmen der Diskussion ein allgemeiner Austausch über Modelle, Möglichkeiten und Realitäten der Abfallsammlung und -entsorgung statt.

Mit einer Danksagung des Institutsdirektors Durner an den Referenten und die Teilnehmer der Tagung schloss das 334. Wasserrechtliche Kolloquium, das mit seinem kreislaufwirtschaftsrechtlichen Inhalt die konstruktiv-kritische Begleitung des Wertstoffgesetzes auf seinem Werdegang seitens des IRWE fortsetzte.

\title{
BUCHBESPRECHUNGEN
}

DOI: 10.1007/s10357-016-3109-5

\section{Bernhard Stüer/Willi Esch Probstfeld, Die Planfeststellung. Grundlagen - Fachrecht - Rechtsschutz - Beispiele}

\section{Aufl. 2016. XXVII, 887 Seiten, Hardcover. Verlag}

C.H. Beck, München. 99,00 Euro, ISBN 978-3-406-69429

Die Planfeststellung ist das zentrale Institut des Fachplanungsrechts und ein wichtiges Instrument für die Verwirklichung von Infrastrukturprojekten. Bei derartigen - vielfach hochkomplexen - Vorhaben können das Planfeststellungsverfahren mit seiner umfassenden Behörden-, Verbände- und Öffentlichkeitsbeteiligung sowie der planerische Gestaltungsspielraum der Planfeststellungsbehörde die vielfältigen öffentlichen und privaten Belange ermitteln und in Ausgleich bringen. Komplex sind indes nicht allein die im Wege der Planfeststellung zuzulassenden Vorhaben, sondern vor allem auch die hierfür einschlägigen Rechtsgrundlagen: Die das Planfeststellungsverfahren anordnenden Vorschriften sind auf zahlreiche Fachgesetze verteilt, und auch nach dem Planvereinheitlichungsgesetz (BGBl. I 2013, S. 1388) finden sich dort manche Sondervorschriften; anspruchsvoll sind aber auch die allgemeinen Regelungen der $\$ \$ 72 \mathrm{ff}$. VwVfG. Hinzu kommen die vielfältigen Vorgaben insbesondere des Umweltrechts und der räumlichen Gesamtplanung, die zum Prüfprogramm im Planfeststellungsverfahren gehören. Das erstmals 2003 von Bernhard Stüer und Willi Esch Probstfeld aufgelegte Handbuch „Die Planfeststellung“ will neben einer Gesamtdarstellung des Planfeststellungsrechts auch die in der Praxis gesammelten Erfahrungen der Autoren weitergeben und zielt damit in besonderer Weise auf eine Veranschaulichung des komplexen und vielfach als abstrakt empfundenen Stoffes. Die dynamische Fortentwicklung von Gesetzgebung und Rechtsprechung hat nunmehr eine Neuauflage erforderlich gemacht, die von Stüer allein verantwortet wird.

Gut die Hälfte des Werkes ist den „Grundlagen“ der Planfeststellung gewidmet (Teil A., S. 1-426). Das Kapitel „Rechtsgrundlagen“ zeichnet zentrale Entwicklungslinien in der Gesetzgebung, aber auch der Rechtsprechung zum Planfeststellungsrecht nach. Dem folgt eine umfassende Darstellung des Planfeststellungsverfahrens, insbesondere der Umweltverträglichkeitsprüfung sowie der Behörden- und Öffentlichkeitsbeteiligung. Anschaulich wird die Behandlung von Einwendungen und Stellungnahmen und die Durchführung des Erörterungstermins beschrieben. Eine ausführliche Darstellung erfahren jedoch auch die materiellen Anforderungen an die Planfeststellung, namentlich die Planrechtfertigung, das zwingend zu beachtende Recht und das Abwägungsgebot. Einen Schwerpunkt bildet hier sachgerecht das praktisch überaus bedeutsame Naturschutzrecht, insbesondere das europäische Gebiets- und Artenschutzrecht; doch

Dr. Boas Kümper,

Münster, Deuschland finden auch der Lärmschutz und die Ausgleichsmaßnahmen angemessene Berücksichtigung. Dem Grundlagenteil folgt - gleichsam als „Besonderer Teil“ - ein naturgemäß ebenfalls sehr langer Abschnitt „Fachrecht“ (Teil B., S. 427-825), in welchem die Planfeststellung nach den einzelnen Fachplanungsgesetzen - FStrG, AEG, LuftVG, PBefG, EnWG, NABEG und viele mehr - behandelt wird. Vorangestellt ist der Darstellung der einzelnen Fachrechte ein Kapitel über „Schnittstellen zwischen Bau- und Fachplanungsrecht", welches gemeinsame Strukturen des Fach- und Gesamtplanungsrechts herausstellt und das Zusammenwirken der verschiedenen Planungsentscheidungen behandelt. Anschließend nimmt unter den Fachrechten das Fernstraßenrecht den breitesten Raum ein, was auf den Modellcharakter dieser Materie für das Fachplanungsrecht hindeutet. Hier wird nicht nur die fernstraßenrechtliche Planfeststellung, sondern werden auch die dieser vorgelagerten Entscheidungen der Bedarfsplanung und der Linienbestimmung eingehend dargestellt - zu Recht, sind diese doch für die Planrechtfertigung und die Abwägung auf der Stufe der Planfeststellung von zentraler Bedeutung. Eine eingehende Darstellung erfährt etwa auch das Eisenbahnrecht, wo u.a. das schwierige Verhältnis von Eisenbahnplanung und Bauleitplanung ausführlich behandelt wird. Sehr knapp fallen demgegenüber die Kapitel zum Energieplanungsrecht aus; hier werden überwiegend lediglich die einschlägigen Vorschriften des EnWG und des NABEG im systematischen Überblick referiert, ohne diese einer näheren Analyse zu unterziehen. Ein Abschnitt zum Rechtsschutz im Fachplanungsrecht (Teil C., S. 827-858) rundet das Werk ab. Hier steht naturgemäß die Anfechtung des Planfeststellungsbeschlusses im Vordergrund und gilt das besondere Augenmerk den unionsrechtlichen Einwirkungen auf das nationale Verwaltungsprozessrecht.

Die Darstellung wird dem selbst gesetzten Anspruch auf Anschaulichkeit und Praxisorientierung vollauf gerecht: Zahlreiche Übersichten strukturieren die komplexen Verfahrens- und Prüfungschritte, zentrale Begriffe werden in optisch hervorgehobenen Zusammenfassungen prägnant erläutert, es finden sich zahlreiche Vorschläge zur Umsetzung der rechtlichen Vorgaben in der Planungspraxis. Eher der Auflockerung dienen die zahlreichen fotographischen Abbildungen („Überführung des SuperstarVirgo von Papenburg nach Emshaven“, „Schwarzspecht“, „Bürgerproteste Altrip“" usw.) und der schwungvolle, bisweilen auch leicht ironische Stil, wie er sich etwa in der Bezugnahme auf „die Vögel auf dem Felde“ (Mt 6, 26) niederschlägt. Inhaltliche Kritik lässt sich allenfalls vereinzelt üben: So wird etwa missverständlich in Rdnr. 58 und in der Übersicht bei Rdnr. 65 formuliert, die Plangenehmigung habe - anders als die Planfeststellung keine enteignungsrechtliche Vorwirkung. Seit dem Planvereinheitlichungsgesetz aber wird die enteignungsrechtliche Vorwirkung durch $\$ 74$ Abs. 6 S. 2 VwVfG - anders als nach der alten Fassung der Vorschrift - nicht mehr gesetzlich ausgeschlossen, so dass sie nach Maßgabe des Fachrechts für die Plangenehmigung möglich und auch vielfach angeordnet ist (siehe etwa $\$ 19$ Abs. 2 FStrG, $\$ 45$ Abs. 2 EnWG, \$71 S. 3 WHG). Derartige Details vermögen jedoch nicht, den Gesamtwert des Werkes nennenswert zu mindern. Das Handbuch kann vielmehr allen mit dem Planfeststellungsrecht Befassten wertvolle Anregungen und Hilfestellungen bieten. 